\title{
A computational binding affinity estimation protocol with maximum utilization of experimental data : A case study for Adenosine Receptor
}

\author{
Il Kwon Cho a , Sung Hyun Moon ${ }^{\text {a }}$, and Kwang-Hwi Cho a, b, * \\ ${ }^{a}$ School of Systems Biomedical Science, Soongsil University, Seoul, Republic of Korea. \\ ${ }^{\mathrm{b}}$ AzothBio, Rm. 1306 MState A, 114, Beobwon-ro, Songpa-gu, Seoul, Republic of Korea \\ * Corresponding author. School of Systems Biomedical Science, Soongsil University, Seoul, \\ Republic of Korea
}

E-mail addresses: chokh@,ssu.ac.kr

\begin{abstract}
Estimating binding affinity between a target protein and the ligand is a crucial step in the drug discovery process. In computer-aided drug design (CADD), the problem can be divided into two steps, finding the correct binding pose and estimating binding free energy. In this study, a new binding affinity estimation protocol, which uses molecular docking and binding affinity estimation with Molecular Dynamics (MD) simulation and maximizes the use of available experimental data, is suggested. Docking with a custom scoring function was used to find a better initial binding pose and Linear Interaction Energy (LIE) method with an optimized coefficient was used to estimate the binding affinity. The protocol has been validated with an external validation set and applied to five modafinil and its derivatives to set the order of binding affinity to Adenosine A2A receptors (ADORA2A, A2aR), which is a membrane protein, for a case study. This protocol could be time-efficient and useful for computational drug discovery where limited experimental data is available.
\end{abstract}

Keywords : Computer-Aided Drug Discovery; Binding poses; Binding Affinity; Adenosine Receptor; Modafinil; Molecular Dynamics Simulation

\section{Introduction}

With growing knowledge of Computer-Aided Drug Design (CADD) and computational power, CADD has been widely used and developed in the pharmaceutical industry to alleviate difficulties in classical drug discovery. [1,2] Examples of technologies performed in CADD include Molecular Dynamics (MD) simulation, quantitative structure-activity relationship (QSAR), pharmacophore modeling, and molecular docking [3]. Among them, molecular docking is commonly used in virtual screening and the choice of docking pose as 
fast method [4,5], whereas it lacks accuracy and shows substantial difference between the pose applying docking and the final pose produced by MD simulation [6]. For this reason, Molecular docking is used to screen possible candidates from the huge chemical database rather than finding the final pose and affinity. More accurate pose and affinity could be estimated by MD simulation and binding free energy calculation [7,8]. Experimental measurements of protein-ligand interactions are measured by $\mathrm{Ki}, \mathrm{Kd}$, and IC50 which are an average value of huge ensemble $[9,10]$. However, the counter part of those values in CADD is binding free energy and very difficult to estimate computationally with the desired accuracy.

Estimating binding free energy can be divided into two parts. One is finding a reasonable binding pose and another is the calculating binding free energy of the complex. Initial binding poses are frequently estimated by docking study if there is no available experimental evidence [11]. The molecular docking technique is an efficient method to screen possible candidates from a huge database, however, the lowest energy pose does not correspond to its experiments frequently $[12,13]$. One of the ways of overcoming the shortcomings is considering multiple initial poses. However, there is no golden rule for the number of initial poses to be considered, and considering more complex structures means more computational time.

Second challenge for binding free energy estimation is the calculation of the binding free energy itself. Calculating binding free energy is one of the crucial goals in CADD, where lots of methods have been developed and refined in terms of a time-consuming process. For example, one of the rigorous alchemical free energy calculations, free energy perturbation (FEP) $[14,15]$ which is based on physical effective energy functions, is computationally very expensive. Less expensive methods like Molecular Mechanics Poisson-Boltzmann Surface Area (MMPBSA)[16,17] and linear interaction energy (LIE) [18,19], which are end-point methods employing the bound and unbound states of protein-ligand, are utilized as alternatives. However, when it comes to estimating configurational entropy of the protein and ligand using normal mode analysis in the MMPBSA method because it requires huge computational time for a single configuration and yields uncertain results, it shows lower accuracy than FEP $[20,21,22]$. In the LIE method, it gave improved results when relevant binding poses and experimental values of the protein-ligand complex were provided [23].

All the free energy calculation methods mentioned above require long MD simulation. Theoretically, MD simulation could allow the ligand to move to find its proper position and orientation in the binding site if the simulation time is long enough. However, in practice, a bad initial structure frequently gives inaccurate results. Starting from a good initial structure is a critical point in estimating binding free energy.

G-protein-coupled receptor (GPCR) plays a vital role in drug discovery, where it takes approximately $27 \%$ of curative drugs in the global market [24]. However, as there are still many challenges in the crystallization of membrane receptors [25,26,27], such as the restricted availability of detergents, it is inevitable to explore drug targets applying CADD. 
Adenosine A2A (ADORA2A, A2aR) receptors are included in the adenosine receptor group of G-protein-coupled receptor (GPCR), where its downregulations of biochemical mechanisms, such as interaction with hormones and neurotransmitters [28,29,30], contribute to excavating drug candidates. Many studies have shown that caffeine and ADORA2A have a strong correlation that caffeine acts as an antagonist to affect sleep deprivation $[31,32,33]$. However, modafinil, which has the same effect on sleep such as caffeine, lacks studies concerning the interaction with ADORA2A. Adenosine A2A receptor was used as a case study to test the protocol suggested.

In this paper, we present a binding affinity calculation protocol that finds better initial poses by using custom scoring and calculates binding free energy with the LIE method which standardized existing experimental data. This protocol is designed to take maximize advantage from currently available experimental data for finding an initial binding pose and calculating binding affinity. The setup procedure for the protocol, validating with external data, and applying to modafinil and its derivates for Adenosine receptors are described in the following sections.

\section{Material and Methods}

\subsection{Protein structure preparation}

The crystal structure of the human Adenosine A2A receptor (ADORA2A, A2aR) was obtained from the RCSB Protein Data Bank (PDB: 3EML) [34] and its missing residues between PRO149 and HIS155 were generated as an unstructured loop of 7 residues using MODELLER [35].

\subsection{Preparation of ligand structure and experimental binding affinity}

Compound T1 to T6 were used to construct the protocol and compound V1, V2 [36,37] were employed for validating the protocol for the ADORA2A receptor. (Figure 1 and Table 1 ). Compound M1 to M5 ( Figure 1) were used for a test study. The experimental binding free energy presented in the Exp column in Table 1 was derived from the inhibition constant (Ki) with the following equation (1) [38,39].

$$
\Delta \mathbf{G}_{\text {exp }}=\mathbf{R T} \ln (\mathrm{Ki})
$$

Where, $\mathrm{R}$ and $\mathrm{T}$ represents the gas constant and the temperature respectively, and the $\mathrm{Ki}$ indicates the concentration for producing $50 \%$ of maximum inhibition. Two compounds, V1 and $\mathrm{V} 2$, are chosen as an external validation set because they have stronger binding affinity than compound T1 to T6. Compound M1 to M5, which are modafinil (M1) and its derivatives (M2 to M5), were used without any experimental binding affinity values were 
used for a case study. (Figure 1 and Table 1) Relative binding orders were estimated with the protocols. All ligand structures were obtained from the PubChem [40] database in SDF format.

Visualization and hydrogen editing of all ligand structures were analyzed in Chimera (v1.14) [41]. Additionally, their approximate partial charges were computed based on AM1BCC (Austin Model 1 with Bond and Charge Correction) in the Antechamber module [42] for MD simulation.

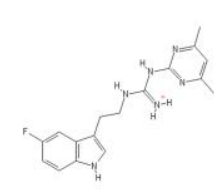

$\mathrm{T} 1$

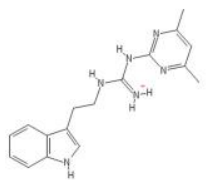

$\mathrm{T} 2$

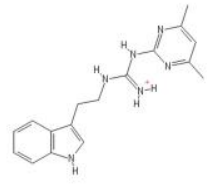

T3

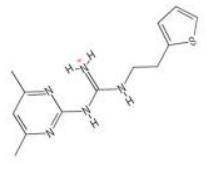

T5

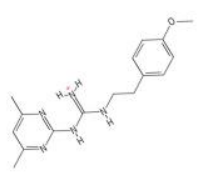

T6
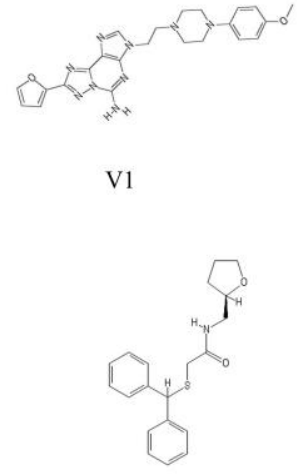

M2

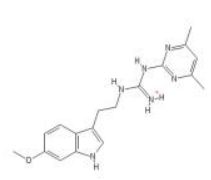

T4

西




\begin{tabular}{|c|c|c|c|c|c|}
\hline T1 & -9.50 & -7.45 & -21.17 & -21.17 & -10.00 \\
\hline $\mathbf{T} 2$ & -9.08 & -7.24 & -16.33 & -18.77 & -9.18 \\
\hline T3 & -8.83 & -7.88 & -23.33 & -16.06 & -7.89 \\
\hline T4 & -7.42 & -6.78 & -15.08 & -15.08 & -6.75 \\
\hline T5 & -9.08 & -6.16 & -16.80 & -16.80 & -9.26 \\
\hline T6 & -8.58 & -6.96 & -16.45 & -17.87 & -9.05 \\
\hline V1 & -14.18 & -8.56 & & -16.87 & -13.86 \\
\hline $\mathbf{V} 2$ & -16.07 & -7.11 & & -17.31 & -13.99 \\
\hline M1 & & & & -11.45 & -9.58 \\
\hline M2 & & & & -20.04 & -13.30 \\
\hline M3 & & & & -19.15 & -11.58 \\
\hline M4 & & & & -14.46 & -9.85 \\
\hline M5 & & & & -15.98 & -13.20 \\
\hline
\end{tabular}

\subsection{Custom scoring with Smina}

Molecular docking experiments were implemented using AutoDock Vina 1.1.2 [44] (Vina) and Smina [45] respectively. In docking preparation steps, missing hydrogens atoms in the modeled structure of the $\mathrm{A} 2 \mathrm{aR}$ receptor were added considering hydrogen bonds with Chimera (v1.14). Energy minimization and file formats conversions of ligands were carried out through RDkit software [46]. The grid box encircling the ligand-binding site is defined large enough to accommodate other A2aRs from the PDB database (PDB: 2YDO, 2YDV, 3PWH, 3REY, 3RFM, 3UZA, 3UZC, and 6GT3) and aligned to the pre-equilibrated modeled-protein for custom scoring with Smina.

Custom scoring function with Smina was obtained based on the training data set that consists of 890 compounds, where 495 compounds obtained from the PubChem database indicating ligands are actively bound to $\mathrm{A} 2 \mathrm{aR}$ and their 495 inactive decoys were generated in Directory of Useful Decoys-Enhanced (DUD-E) database [47]. The performance of the improved custom scoring function was shown in Figure 2. The AUC of the custom scoring function has been improved from 0.656 to 0.779 . 


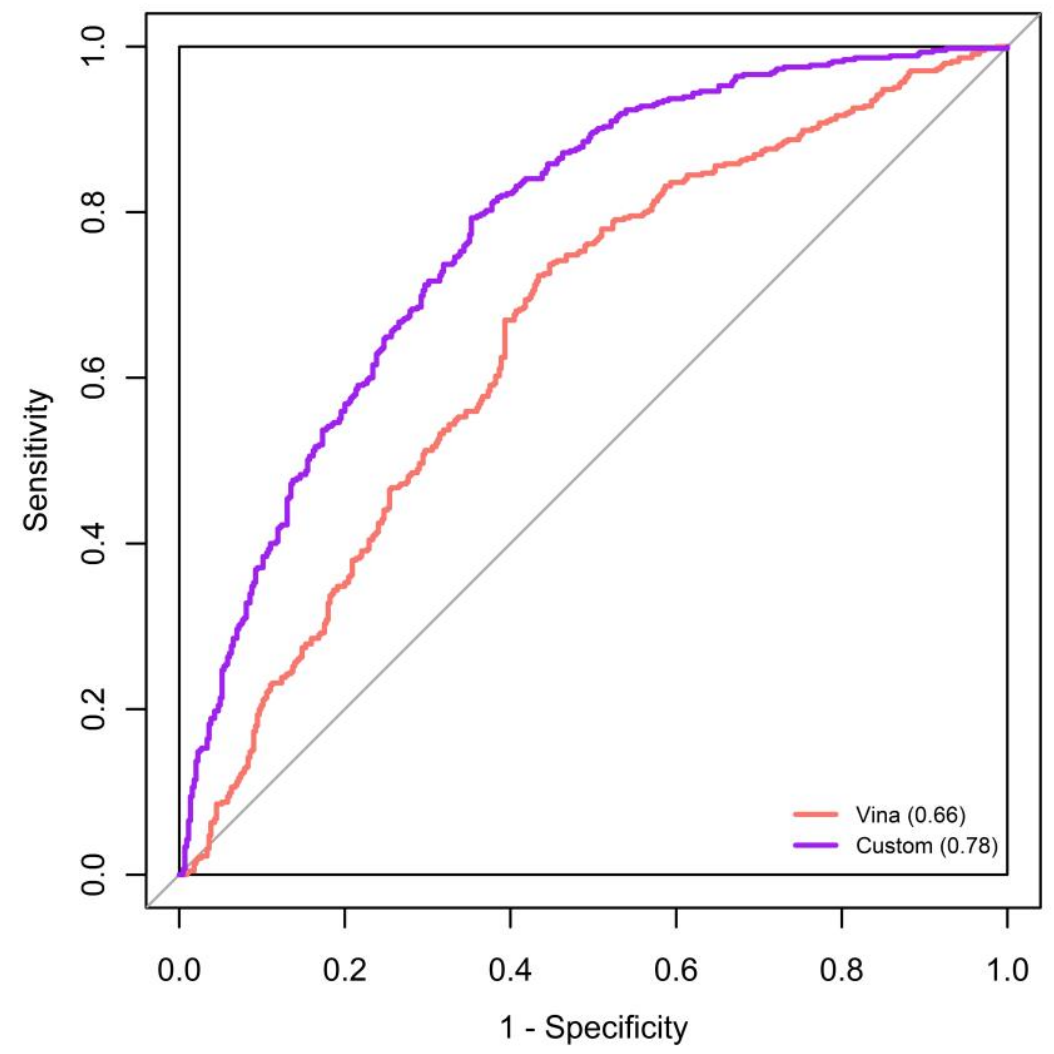

Fig. 2. The performance of the improved custom scoring function

20 different binding poses for each compound were generated with Vina. Among them, three top-ranked poses indicating the highest binding affinity were selected as the representatives of Vina. Then, the custom scoring function was applied to the 20 poses to select the top-ranked pose to make the representative of custom scoring with Smina. The selected poses, three from Vina and one from custom scoring were used for the initial poses of following MD simulations. The first ranked poses of custom scoring function for compound T1 to T6 were originally ranked as 3, 4, 14, 2, 2 and 6 of Vina for each compound, respectively.

\subsection{Molecular Dynamics Simulations}

In this protocol, the membrane systems of all models were constructed using membrane builders in CHARMM-GUI $[48,49]$. All models were inserted in 100\% 1-palmitoyl-2-oleoylsn-glycero-3-phosphocholine (POPC) bilayers and the bilayers were oriented based on the 
Position of Proteins in Membrane (PPM) server [50]. Models were solvated and ionized of $0.15 \mathrm{M} \mathrm{NaCl}$ concentration. A rectangular lipid bilayer of $\sim 95 \times 95 \AA^{2}$ was constructed considering $\mathrm{x}, \mathrm{y}$ dimensions of membrane plane. Compositions of the initial protein-only membrane model were described in Table S1 and Figure S1.

All equilibration and production MD simulations were performed with NAMD 2.13 software [51] and VMD 1.9 software [52]. The parameters of lipids and the protein were generated by applying the CHARMM36 force field [53] and their ligands were generated with the CGenFF server [54]. In this work, we follow the equilibration protocol with bond constraints and harmonic restraints suggested in CHARMM-GUI which was postulated previously in membrane system setup. Briefly, equilibration simulations were composed of six steps; energy minimization and equilibration steps in the NVT ensemble with positional restraints were carried out and shortly after equilibration steps proceeded in the NPT ensemble by weakening the position restraints of protein and membrane atoms sequentially in the following steps. Next, production simulations were conducted by removing all restraints applied to atoms.

Three replication simulations applied to each pose were processed. All simulations with positional restraints were run for $6.77 \mathrm{~ns}$. Before running the protein-ligand complex system, the $\mathrm{A} 2 \mathrm{aR}$ receptor was pre-equilibrated with the same equilibration and production steps. All bonds applied to hydrogen atoms in all simulations were constrained using the SHAKE algorithm [55]. The value switch distance of van der Waals potential energy was set to $10 \AA$, the point that started to reduce smoothly for preserving function continuously and became 0 at $12 \AA$ with cutoff distance in all simulations. Additionally, PME (Particle Mesh Ewald) method [56] was employed for computing the long-range interactions in periodic boundary conditions.

After equilibration steps, a time step of 2 fs was conducted in production simulations and snapshots were saved every $100 \mathrm{ps}$ in NAMD ded trajectories. In production simulations, NPT simulations were run; Temperature was maintained at $310 \mathrm{~K}$ by the Langevin dynamics with a coupling coefficient of $1 p^{-1}$ and the pressure was upheld at $1.01325 \mathrm{bar}(1 \mathrm{~atm})$ by the Langevin piston Nose-Hoover method with the Langevin piston period of 50fs and the Langevin piston decay of 25 fs [57]. All trajectories in ded format were analyzed and visualized through root-mean-square deviations (RMSD). RMSD studies were analyzed and aligned based on seven transmembrane helices surrounded with POPC bilayers of the first equilibration snapshot.

\subsection{Validating the use of Custom Scoring function through MMPBSA.}

Compound T1 to T6 were used for this stage. Each compound produces the three topranked poses from Vina and one top-ranked pose from the custom scoring function. Three 
replicas are considered for each pose which makes a total of 24 protein-ligand poses (18 poses for vina and six poses for custom scoring function). With the MD simulation trajectories, the Molecular Mechanics Poisson-Boltzmann Surface Area (MMPBSA) method is applied to get binding free energy.

The binding free energy equation of protein-ligand complex in the MMPBSA method was illustrated as :

$$
\begin{gathered}
\Delta \mathbf{G}_{\text {bind }}=\boldsymbol{G}_{\text {complex }}-\boldsymbol{G}_{\text {receptor }}-\boldsymbol{G}_{\text {ligand }} \text { (2) } \\
\Delta \mathbf{G}_{\text {bind }}=\Delta \mathbf{E}_{M M}+\Delta \boldsymbol{G}_{\text {bind,solv }}-\mathrm{T} \Delta \mathbf{S} \text { (3) }
\end{gathered}
$$

where $G_{\text {complex }}, G_{\text {receptor }}$, and $G_{\text {ligand }}$ indicate the total free energy in solvent of a protein-ligand complex, $\mathrm{A} 2 \mathrm{aR}$, and each ligand respectively. Those three terms were approximately computed as (3) where $\Delta \mathrm{E}_{M M}$ represents molecular mechanics energy in the gas phase, $\Delta G_{\text {bind,solv }}$ indicates solvation polar energy, and $\mathrm{T} \Delta \mathrm{S}$ indicates the conformational entropy change with ligand binding.

$$
\Delta \mathbf{E}_{M M}=\Delta \mathbf{E}_{c o v}+\Delta \mathbf{E}_{e l e c}+\Delta \mathbf{E}_{v d w}
$$

$\Delta \mathrm{E}_{\mathrm{MM}}$ was separated into covalent bond energy $\left(\Delta E_{\text {cov }}\right)$, electrostatic interaction energy $\left(\Delta E_{\text {elec }}\right)$, and van der Waals interaction energy $\left(\Delta E_{v d w}\right)(4)$ where covalent bonds were offset according to the equation (2).

$$
\begin{aligned}
\Delta \boldsymbol{G}_{\text {bind,solv }} & =\Delta \boldsymbol{G}_{P B}+\Delta \boldsymbol{G}_{n p} \\
\Delta \boldsymbol{G}_{n p} & =\gamma \mathrm{SASA}+\boldsymbol{\beta}
\end{aligned}
$$

In the MMPBSA method, $\Delta G_{\text {bind,solv }}$ was split into electrostatic solvation free energy and non-polar solvation free energy $\left(\Delta G_{n p}\right)$ (5). Electrostatic solvation energy was obtained using the Poisson-Boltzmann implicit solvent model $\left(\Delta G_{P B}\right)$ and non-polar solvation free energy $\left(\Delta G_{n p}\right)$ was computed using the solvent-accessible surface area (SASA) [58] (6). Constant values of $0.00542 \mathrm{kcal} /\left(\mathrm{mol} \cdot \AA^{2}\right)$ and $0.92 \mathrm{kcal} / \mathrm{mol}$ were used with the surface tension $(\gamma)$ and the correction term $(\beta)$ in equation (6) respectively.

All the equations of MMPBSA were implemented using the Calculation of Free Energy (CaFE) [59] in the VMD plugin and the Poisson-Boltzmann calculation model was solved through the Advanced Poisson-Boltzmann Solver (APBS3.0) [60]. T $\Delta \mathrm{S}$ denotes the change in conformational entropy of protein-ligand interaction (3). This term can be calculated through normal-mode analysis. However, as this approach is computationally demanding and tends to draw high standard deviations among MD simulations' models caused by small snapshots and the purpose of using MMPBSA was to compare the performance of Smina to Vina, the term was ignored.

These calculations were performed to show that using the custom scoring function with Smina is superior to Vina for finding a better initial pose. All the production simulations were divided into two steps by evaluating the consistency of fluctuation with RMSD values. 500 
frames representing 50ns of production simulations as we saved snapshots at an interval of 100 ps (Figure S2) were analyzed for calculating binding free energies applying the MMPBSA method.

The correlation coefficient between Vina and experimental values is 0.2407 which is very poor ( Figure 3(a) ). When the best scored pose from Vina was used to calculate MMPBSA energy, the correlation between MMPBSA energy and experimental values was 0.2428 (Figure 3(b) ) which is not much improved from the Vina score. When the top three ranked poses are considered, the correlation coefficient was 0.5118 ( Figure 3(c)). It clearly shows that using a proper initial pose is more important than the choice of a free energy calculation method.

The best MMPBSA results from three poses from Vina and that of one pose from custom scoring are represented in the column of Vina + MMPBSA and Smina + MMPBSA in Table 1, respectively. All values for 24 models including replicas are represented in Table S2. The data shows that MMPBSA energy from the initial pose from custom scoring finds lower energy complex than that from Vina except for the case of compound T3, even though it considers only one best pose. In addition, when it comes to average binding energy of three replications, custom scoring models present the best pose except the compound T3. (Table S2). The Pearson's correlation coefficients between the best MMPBSA energy and experiments were 0.5118 for Vina and 0.7685 for Smina. ( Figure 3 ). Even though Smina considered only one best pose with three replicas, it shows superior results to vina with three poses with 9 replicas for each compound. It could be concluded that custom scoring function with Smina shows better results than vina for finding initial pose for protein-ligand complex.
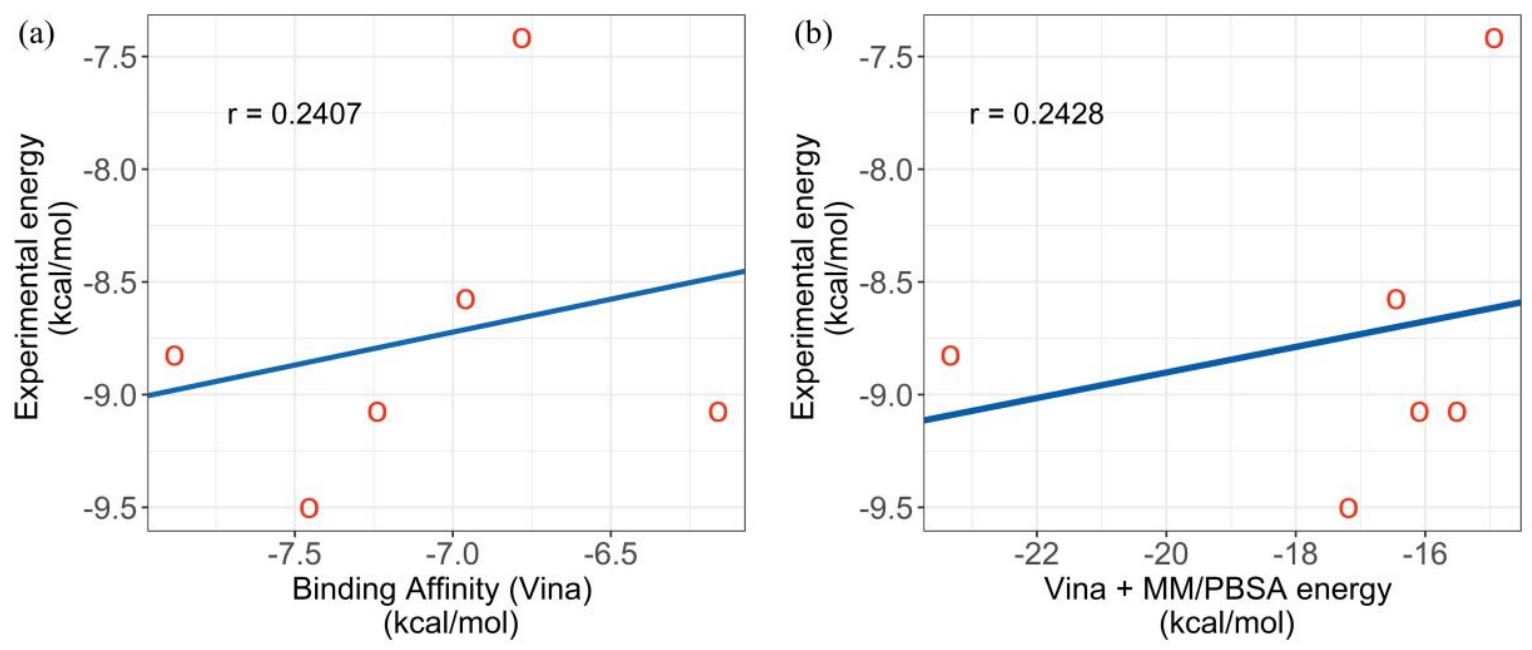

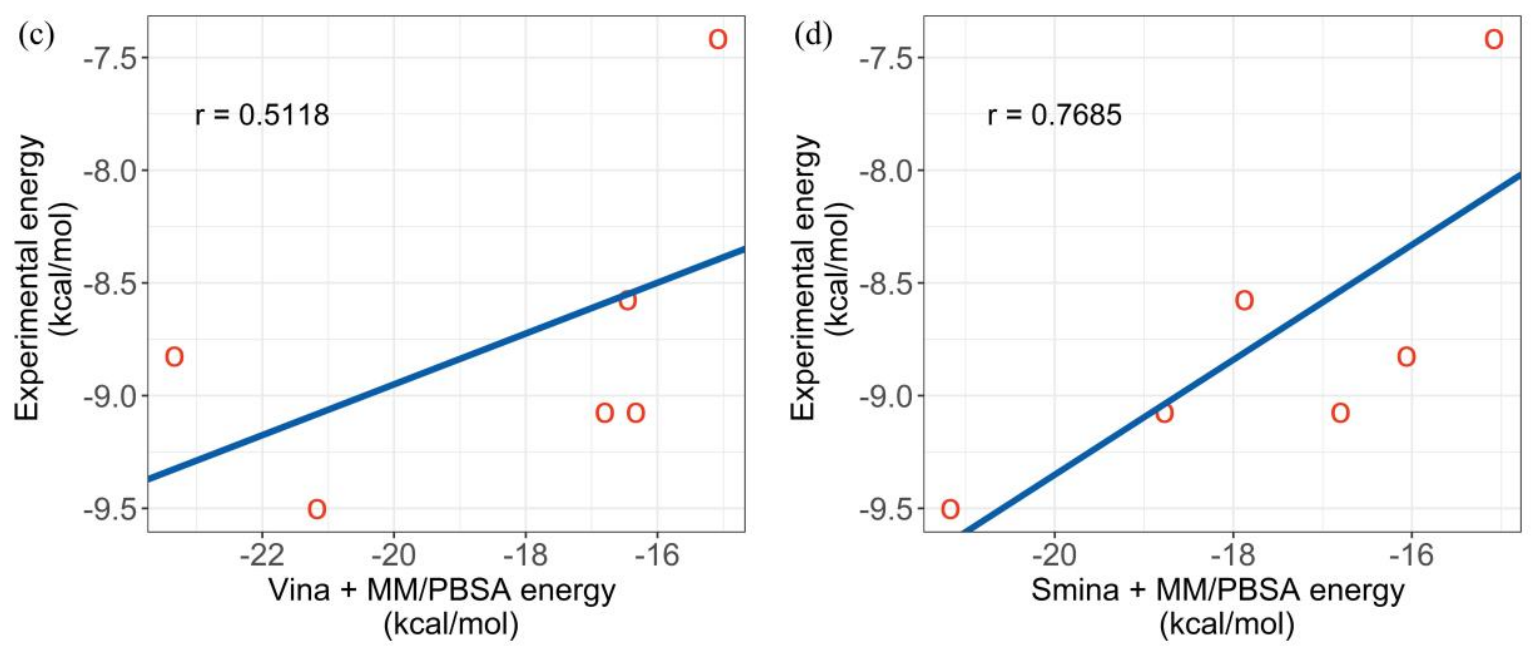

Fig. 3. The Correlation coefficient between experimental and calculated values of (a) the best pose from Vina score, (b) MMPBSA energy with the best pose from Vina, (c) the best MMPBSA energy from top three ranked poses from Vina, and (d) the best MMPBSA energy from top-ranked pose from Smina.

\subsection{Determination of coefficients for LIE method}

At the final stage, the linear interaction energy (LIE) method with known experimental data is considered to improve the accuracy of the protocol. LIE, the alternate end-point binding free energy calculation, was used in our protocol.

$$
\begin{aligned}
& \Delta G_{\text {bind }}=\alpha\left(\left\langle V_{\text {lig-surr }}^{\text {vdw }}\right\rangle_{\text {bound }}-\left\langle V_{\text {lig-surr }}^{\text {vdw }}\right\rangle_{\text {unbound }}\right) \\
& +\beta\left(\left\langle V_{\text {lig-surr }}^{\text {elec }}\right\rangle_{\text {bound }}-\left\langle V_{\text {lig-surr }}^{\text {elec }}\right\rangle_{\text {unbound }}\right)
\end{aligned}
$$

As illustrated in equation (7), $\Delta \boldsymbol{G}_{\boldsymbol{b i n d}}$ was analyzed by collaborating two parameters assumed to be related linearly where van der Waals and electrostatic energy were utilized as parameters. Van der Waals and electrostatic energy denote the differences between averaged bound state and unbound state energies in the solvent system respectively. Each state was obtained through interaction energies between the ligand (lig) and the surroundings of the ligand (surr). $\boldsymbol{\alpha}$ and $\boldsymbol{\beta}$ in equation (7) represent the coefficients of Van der Waals and electrostatic energy, respectively. Converted experimental Ki values of compound T1 to T6 were utilized as $\Delta \boldsymbol{G}_{\boldsymbol{b i n d}}$ with equation (1). Optimization for the coefficients for LIE models was performed and analyzed using $\mathrm{R}$ software (4.0.0) [61] to minimize the error between 
experimental values and LIE. Optimized coefficients for $\boldsymbol{\alpha}$ and $\boldsymbol{\beta}$ were in equation (7) were 0.317 and 0.034 , respectively. With the optimized coefficients, the LIE model shows 0.553 (kcal/mol) Root-Mean-Square Error (RMSE) and significant R-squared coefficient of determination $\left(R^{2}=0.996\right)$ to the experimental values.

\section{Results and Discussion}

\section{Suggested protocol}

The protocol has been finalized as the following steps.

Step 1.20 binding poses are generated with AutoDock Vina

Step 2. The 20 poses are reordered with a custom scoring function from Smina and the best pose(s) are selected.

Step 3. MD simulation allowing three replicas for the selected pose(s) is performed to get 500 frames of trajectories from $50 \mathrm{~ns}$ simulation.

Step 4. The binding free energy is calculated using the LIE method with the MD trajectory and optimized LIE coefficients.

The protocol is established with compound T1 to T6 in Table 1.

Compound V1 and V2 in Table 1 were used to validate the protocol. After following the protocol, each compound indicated -13.86 and $-13.99 \mathrm{kcal} / \mathrm{mol}$ of binding free energies for V1 and V2, respectively (Table 1). This finding supports that compound V1, V2 bind better than compound $\mathrm{T} 1$ to $\mathrm{T} 6$.

The Pearson's correlation coefficients with experimental values for the protocol ( Smina + LIE column in Table 1) were 0.9037 and 0.9647 for a train set and train + validation set, respectively ( Figure 4 ). In contrast, the Pearson's correlation coefficient with experimental values for Vina only ( Vina column in Table 1 ) were 0.24 , and 0.41 for a train set and train + validation set respectively. 

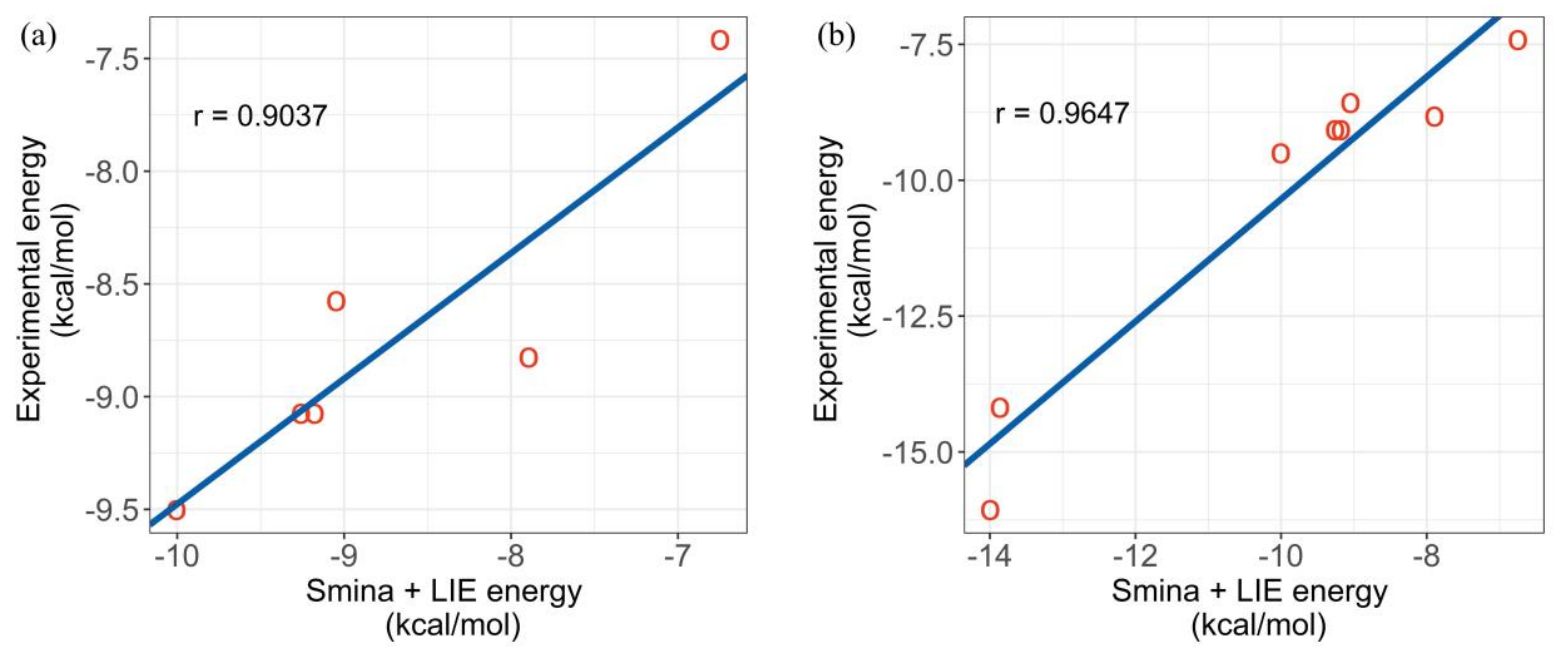

Fig.4. Comparison between LIE energy from this work and experimental data (a) with train set (b) with train + validation set

In order to find new inhibitors for $\mathrm{A} 2 \mathrm{aR}$, modafinil and its derivatives are investigated with the protocol. We considered modafinil as a possible candidate for A2aR inhibition, because an initial docking study with modafinil in the ENAMINE [62] database showed that modafinil (M1) could be a possible lead compound for A2aR inhibition. Four modafinil derivatives were generated (M2 to M5). The modafinil and its derivatives were used further with the protocol to investigate, 1) could they be better inhibitors than any of the known inhibitors, and 2) how are the order of binding affinity for them. The interactions between modafinil and A2aR were not known. First, initial ligand binding poses between A2aR and compounds were obtained with Vina and rescored them with a custom scoring function from Smina. One top-ranked pose was considered with three replicas for MD simulation. As summarized in Table 1, M2 shows the best binding affinities with MMPBSA and LIE method and $\mathrm{M} 2$ is a better binder than modafinil (M1). We found that the order of binding affinity for the compounds is M2>M3 $>$ M5 $>$ M4 $>$ M1. The exact values predicted by the

protocol are tabulated in Table 1. Furthermore, as illustrated in Figure 5, the Pearson's correlation coefficient of 0.7568 shows a positive correlation between MMPBSA energy and LIE energy. We expect that M2 could be a good candidate for the A2aR inhibitor. 


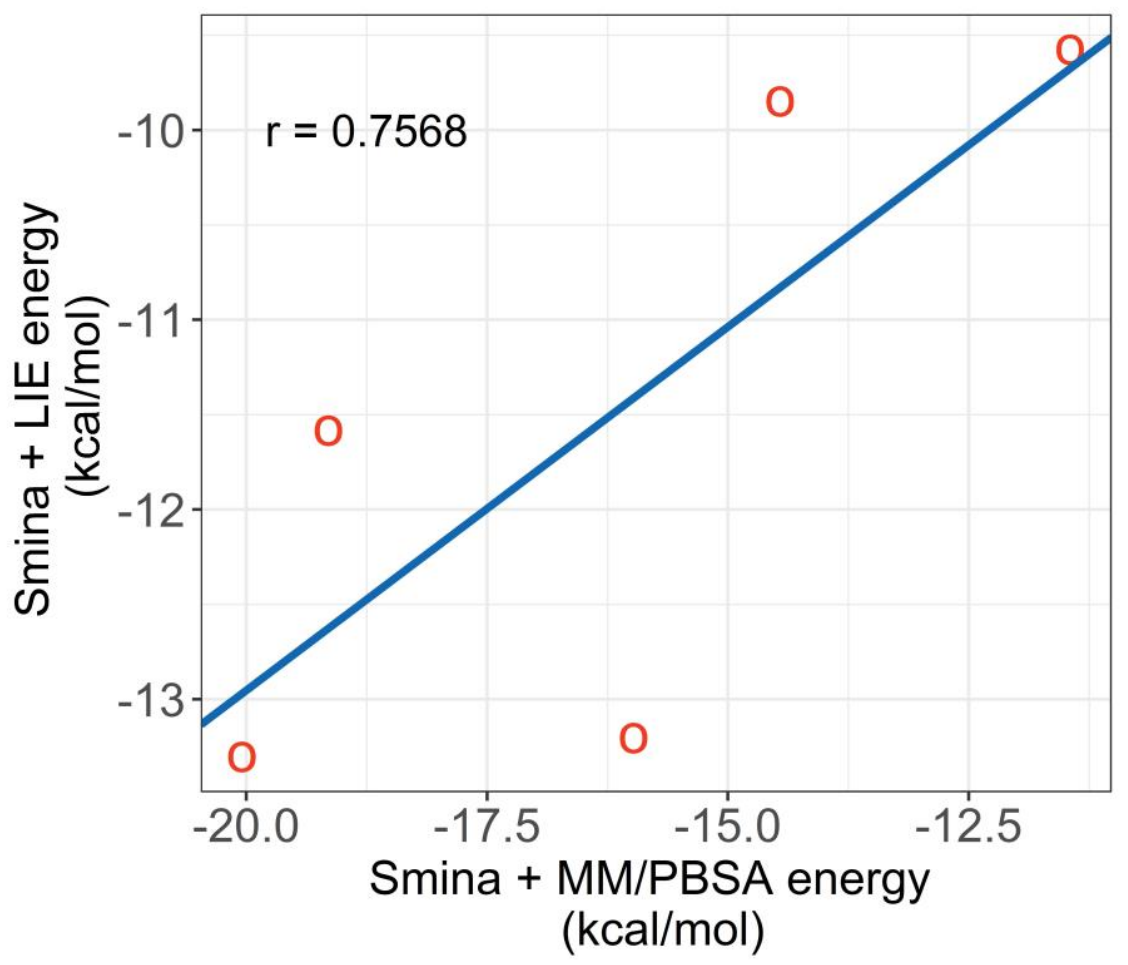

Fig. 5. Correlation between calculated MM/PBSA and LIE energy for compound M1 to M5.

We further investigate the interaction between $\mathrm{A} 2 \mathrm{aR}$ and ligand by looking at the interaction maps. Since none of the structural information between A2aR and modafinil and its derivatives are available. It is difficult to conclude any structural details, however, since the custom scoring function provides better initial structures and MD simulation could allow the ligands to adapt its orientation well in the binding pocket, we performed structural investigation with an interaction map from LigPlot+ (v2.2) [63] (Figure 6). M2 shows more interactions than M1 with residues surrounding. Both ligands consist of two parts, polar and non-polar parts, divided by sulfur atoms in the middle. For the polar part, M2 is surrounded by five residues, CYS339, TYR416, GLU167, MET415, and LEU142, and makes a hydrogen bond with Asn398, while M1 is surrounded by only one residue, CYS390, and makes hydrogen bonding with SER422. For the non-polar part, phenyl of M2 is surrounded by four hydrophobic residues, LEU87, ALA79, VAL170, and VAL182, while that of M1 is surrounded by only two, PHE166 and ALA 57. The binding affinity difference between M1 and M2 could be clearly explained by not only the numerical values but also interaction maps. 

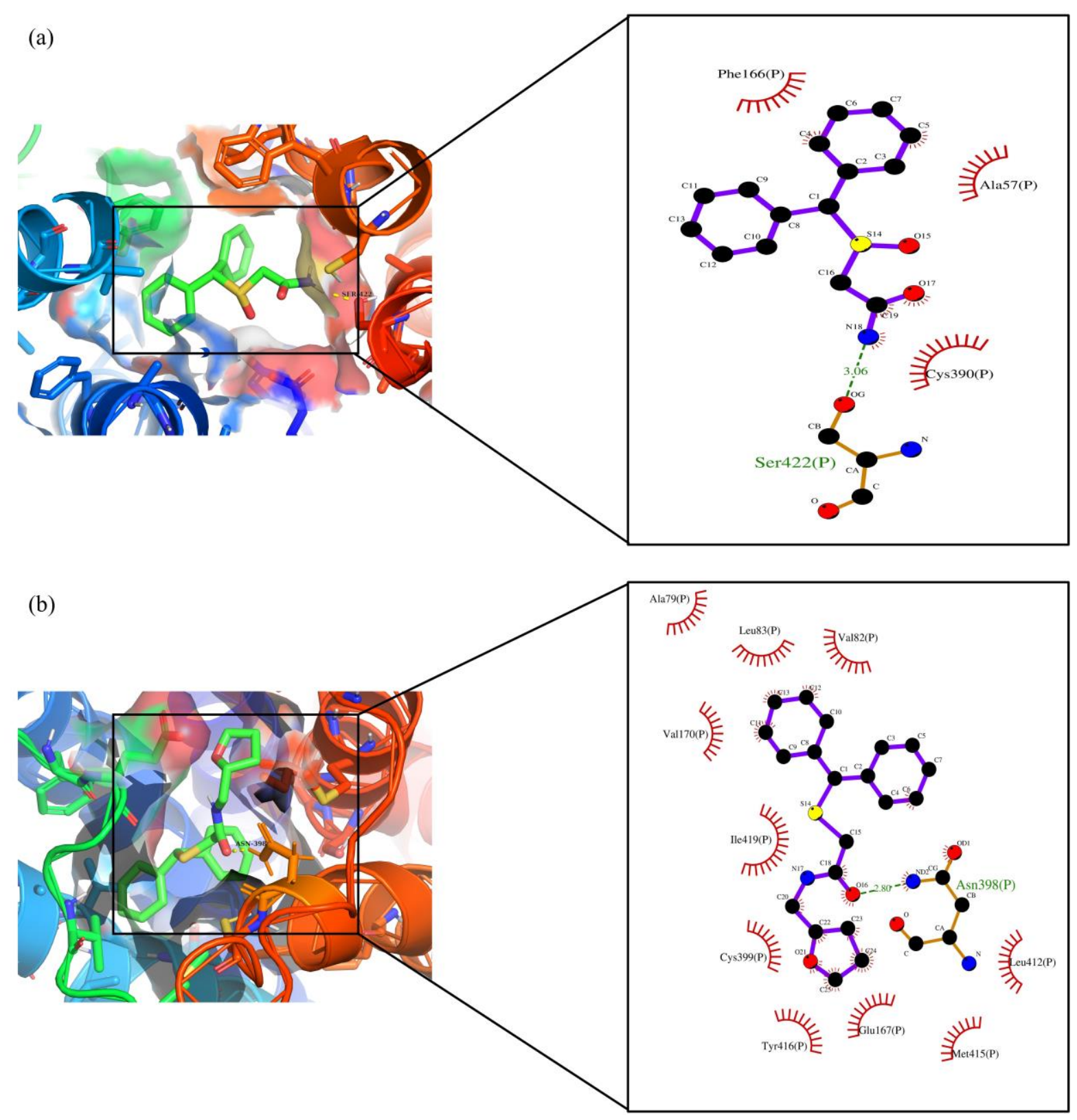

Fig. 6. Interaction map between A2aR and (a) compound M1 and (b) compound M2

Only one binding pose in the Step 2 is considered for the protocol setup procedure and for the case study. However, in Step 2, more than one pose could be considered when more computational resources are available and that could make the result more reliable. However, the protocol found the lowest energy complex structures for five out of six test cases successfully with the train set. The protocol shows much better results than Vina + MMPBSA method which considered three poses for each case. 


\section{Conclusion}

Computer-aided drug design becomes a promising technique for drug discovery as the computing power and methods are developed especially for a membrane protein which is exceedingly difficult to perform experiment with it. One of the main challenges in CADD is predicting binding free energy. Accuracy of predicted binding energy depends on the initial binding pose and the method of choice for binding free energy calculation. The initial binding pose can be guessed by molecular docking, however the scoring function used is developed for general purpose, not for the specific case, and frequently lead to a big deviation from $\mathrm{x}$ ray crystallographic data. The shortage could be overcome by considering not only the best scored pose but also other poses. This strategy also has a limitation. There is no simple rule for the number of poses to be considered and as the number of initial poses increased, the number of the MD simulation, which is the most time-consuming step, is increased as well. In order to solve this problem, a custom scoring function has been introduced and showed that it gives better results than Vina by comparing binding free energy with MMPBSA. For calculation of binding free energy, MMPBSA and LIE methods require less computation time than FEP and any other statistical mechanical method. Among them, LIE utilizes any experimental data available and allows to average large ensemble from long MD simulation. The coefficients for LIE were determined with six experimental data with the correlation coefficient of 0.9037 . The protocol has been validated with two strong binder, compound V1 and V2 show that those compounds are stronger binder than compound T1 to T6. The protocol was applied to Modafinil and its derivatives and found that one of the derivatives (M2) showed the strongest binding affinity than any other compounds considered in this study. This protocol maximizes the use of any available experimental data and is an advanced computational tool for CADD. The protocol could help to accelerate the drug discovery process.

\section{Declaration of competing interests}

The authors declare no conflicts of interest regarding the publication of this article.

\section{Acknowledgements}

We thank Prof. S. H. Suh at Ewha Womans University for the suggestion of modafinil derivatives. KHC would like to thank KREONET (Korean Research Environment Open NET work) which is managed and operated by KISTI (Korean Institute of Science and Technology Information).

\section{References}


[1] D. F. Horrobin, "Innovation in the pharmaceutical industry," J R Soc Med 93, no. 7 (Jul 2000), https://doi.org/10.1177/014107680009300702.

[2] Pratik Swarup Das, Puja Saha, and APJ Abdul, "A review on computer aided drug design in drug discovery," World J. Pharm. Pharm. Sci 6, no. 7 (2017), https://doi.org/10.20959/wjpps20177-9450.

[3] Mohammad Hassan Baig et al., "Computer aided drug design: success and limitations," Current pharmaceutical design 22, no. 5 (2016).

[4] D. B. Kitchen et al., "Docking and scoring in virtual screening for drug discovery: methods and applications," Nat Rev Drug Discov 3, no. 11 (Nov 2004), https://doi.org/10.1038/nrd1549.

[5] Garrett M Morris and Marguerita Lim-Wilby, "Molecular docking," in Molecular modeling of proteins (Springer, 2008).

[6] Y. C. Chen, "Beware of docking!," Trends Pharmacol Sci 36, no. 2 (Feb 2015), https://doi.org/10.1016/j.tips.2014.12.001.

[7] Takefumi Yamashita et al., "The feasibility of an efficient drug design method with high-performance computers," Chemical and Pharmaceutical Bulletin 63, no. 3 (2015), https://doi.org/10.1248/cpb.c14-00596.

[8] C. Pu et al., "Assessing the performance of docking scoring function, FEP, MM-GBSA, and QM/MM-GBSA approaches on a series of PLK1 inhibitors," Medchemcomm 8, no. 7 (Jul 1 2017), https://doi.org/10.1039/c7md00184c.

[9] Cheng Yung-Chi and William H Prusoff, "Relationship between the inhibition constant (KI) and the concentration of inhibitor which causes 50 per cent inhibition (I50) of an enzymatic reaction," Biochemical pharmacology 22, no. 23 (1973), https://doi.org/10.1016/0006-2952(73)90196-2.

[10] Giancarlo Franchini et al., "Ionization and dissociation of weak electrolytes. An initial approach to Ki and Kd evaluation," Analytical Chemistry 62, no. 10 (1990), https://doi.org/10.1021/ac00209a008.

[11] Douglas R Houston and Malcolm D Walkinshaw, "Consensus docking: improving the reliability of docking in a virtual screening context," Journal of chemical information and modeling 53, no. 2 (2013), https://doi.org/10.1021/ci300399w.

[12] K. M. Elokely and R. J. Doerksen, "Docking challenge: protein sampling and molecular docking performance," J Chem Inf Model 53, no. 8 (Aug 26 2013), https://doi.org/10.1021/ci400040d.

[13] T. Pantsar and A. Poso, "Binding Affinity via Docking: Fact and Fiction," Molecules 23, no. 8 (Jul 30 2018), https://doi.org/10.3390/molecules23081899.

[14] Y. Kita et al., "Contribution of the surface free energy perturbation to protein-solvent interactions," Biochemistry 33, no. 50 (Dec 20 1994), https://doi.org/10.1021/bi00254a029.

[15] Peter Kollman, "Free energy calculations: applications to chemical and biochemical phenomena," Chemical reviews 93 , no. 7 (1993), https://doi.org/10.1021/cr00023a004.

[16] Bernd Kuhn et al., "Validation and use of the MM-PBSA approach for drug discovery," Journal of medicinal chemistry 48, no. 12 (2005), https://doi.org/10.1021/jm049081q.

[17] J. M. Swanson, R. H. Henchman, and J. A. McCammon, "Revisiting free energy calculations: a theoretical connection to MM/PBSA and direct calculation of the association free energy," Biophys $J$ 86, no. 1 Pt 1 (Jan 2004), https://doi.org/10.1016/S0006-3495(04)74084-9. 
[18] T. Hansson, J. Marelius, and J. Aqvist, "Ligand binding affinity prediction by linear interaction energy methods," J Comput Aided Mol Des 12, no. 1 (Jan 1998), https://doi.org/10.1023/a:1007930623000.

[19] J. Aqvist and J. Marelius, "The linear interaction energy method for predicting ligand binding free energies," Comb Chem High Throughput Screen 4, no. 8 (Dec 2001), https://doi.org/10.2174/1386207013330661.

[20] Kenneth M Merz Jr, Dagmar Ringe, and Charles H Reynolds, Drug design: structure-and ligand-based approaches (Cambridge University Press, 2010).

[21] MI El-Barghouthi et al., "Free energy perturbation and MM/PBSA studies on inclusion complexes of some structurally related compounds with $\beta$-cyclodextrin," Supramolecular Chemistry 21, no. 7 (2009), https://doi.org/10.1080/10610270802613562.

[22] Nad'a Špačková et al., "Molecular dynamics simulations and thermodynamics analysis of DNA- drug complexes. Minor groove binding between 4 ', 6-diamidino-2-phenylindole and DNA duplexes in solution," Journal of the American Chemical Society 125, no. 7 (2003), https://doi.org/10.1021/ja025660d.

[23] E. A. Rifai et al., "A Comparative Linear Interaction Energy and MM/PBSA Study on SIRT1-Ligand Binding Free Energy Calculation," J Chem Inf Model 59, no. 9 (Sep 23 2019), https://doi.org/10.1021/acs.jcim.9b00609.

[24] The IDG Knowledge Management Center, "Unexplored opportunities in the druggable human genome. The IDG Knowledge Management Center," (2016).

[25] E. P. Carpenter et al., "Overcoming the challenges of membrane protein crystallography," Curr Opin Struct Biol 18, no. 5 (Oct 2008), https://doi.org/10.1016/j.sbi.2008.07.001.

[26] Isabel Moraes et al., "Membrane protein structure determination - the next generation," Biochimica et Biophysica Acta (BBA)-Biomembranes 1838, no. 1 (2014).

[27] Chiara Parravicini et al., "Forced unbinding of GPR17 ligands from wild type and R255I mutant receptor models through a computational approach," BMC structural biology 10, no. 1 (2010), https://doi.org/10.1186/1472-6807-10-8.

[28] Georgina GJ Hazell et al., "G protein-coupled receptors in the hypothalamic paraventricular and supraoptic nuclei-serpentine gateways to neuroendocrine homeostasis," Frontiers in neuroendocrinology 33, no. 1 (2012), https://doi.org/10.1016/j.yfrne.2011.07.002.

[29] Katherine M Betke, Christopher A Wells, and Heidi E Hamm, "GPCR mediated regulation of synaptic transmission," Progress in neurobiology 96, no. 3 (2012), https://doi.org/10.1016/j.pneurobio.2012.01.009.

[30] C. D. Hanlon and D. J. Andrew, "Outside-in signaling--a brief review of GPCR signaling with a focus on the Drosophila GPCR family," J Cell Sci 128, no. 19 (Oct 1 2015), https://doi.org/10.1242/jcs.175158.

[31] JV Rétey et al., "A genetic variation in the adenosine A2A receptor gene (ADORA2A) contributes to individual sensitivity to caffeine effects on sleep," Clinical Pharmacology \& Therapeutics 81, no. 5 (2007), https://doi.org/10.1038/sj.clpt.6100102.

[32] S. Bodenmann et al., "Polymorphisms of ADORA2A modulate psychomotor vigilance and the effects of caffeine on neurobehavioural performance and sleep EEG after sleep deprivation," Br J Pharmacol 165, no. 6 (Mar 2012), https://doi.org/10.1111/j.1476-5381.2011.01689.x. 
[33] Rafael Almeida Nunes et al., "The association between caffeine consumption and objective sleep variables is dependent on ADORA2A c. 1083T> C genotypes," Sleep Medicine 30 (2017),

https://doi.org/10.1016/j.sleep.2016.06.038.

[34] Veli-Pekka Jaakola et al., "The 2.6 angstrom crystal structure of a human A2A adenosine receptor bound to an antagonist," Science 322, no. 5905 (2008), https://doi.org/10.1126/science.1164772.

[35] Benjamin Webb and Andrej Sali, "Comparative protein structure modeling using MODELLER," Current protocols in bioinformatics 54, no. 1 (2016), https://doi.org/10.1002/0471250953.bi0506s15.

[36] Monica Mantri et al., "2-Amino-6-furan-2-yl-4-substituted nicotinonitriles as A2A adenosine receptor antagonists," Journal of medicinal chemistry 51, no. 15 (2008), https://doi.org/10.1021/jm701594y.

[37] Chandra Bhushan Mishra et al., "Design and synthesis of (4E)-4-(4-substitutedbenzylideneamino)-3substituted-2, 3-dihydro-2-thioxothiazole-5-carbonitrile as novel A2A receptor antagonists," Bioorganic \& medicinal chemistry 21, no. 19 (2013), https://doi.org/10.1016/j.bmc.2013.07.005.

[38] Masahiko Tatsumi et al., "Pharmacological profile of antidepressants and related compounds at human monoamine transporters," European journal of pharmacology 340, no. 2-3 (1997), https://doi.org/10.1016/S0014-2999(97)01393-9.

[39] N. Basilio and F. Pina, "Chemistry and Photochemistry of Anthocyanins and Related Compounds: A Thermodynamic and Kinetic Approach," Molecules 21, no. 11 (Nov 10 2016), https://doi.org/10.3390/molecules21111502.

[40] S. Kim et al., "PubChem 2019 update: improved access to chemical data," Nucleic Acids Res 47, no. D1 (Jan 8 2019), https://doi.org/10.1093/nar/gky1033.

[41] E. F. Pettersen et al., "UCSF Chimera--a visualization system for exploratory research and analysis," $J$ Comput Chem 25, no. 13 (Oct 2004), https://doi.org/10.1002/jcc.20084.

[42] Araz Jakalian, David B Jack, and Christopher I Bayly, "Fast, efficient generation of high-quality atomic charges. AM1-BCC model: II. Parameterization and validation," Journal of computational chemistry 23, no. 16 (2002), https://doi.org/10.1002/jcc.10128.

[43] J. Carlsson et al., "Structure-based discovery of A2A adenosine receptor ligands," J Med Chem 53, no. 9 (May 13 2010), https://doi.org/10.1021/jm100240h.

[44] Oleg Trott and Arthur J Olson, "AutoDock Vina: improving the speed and accuracy of docking with a new scoring function, efficient optimization, and multithreading," Journal of computational chemistry 31, no. 2 (2010), https://doi.org/10.1002/jcc.21334.

[45] D. R. Koes, M. P. Baumgartner, and C. J. Camacho, "Lessons learned in empirical scoring with smina from the CSAR 2011 benchmarking exercise," J Chem Inf Model 53, no. 8 (Aug 26 2013), https://doi.org/10.1021/ci300604z.

[46] Greg Landrum, "RDKit: Open-source cheminformatics," http://www.rdkit.org, no. http://www.rdkit.org.

[47] M. M. Mysinger et al., "Directory of useful decoys, enhanced (DUD-E): better ligands and decoys for better benchmarking," J Med Chem 55, no. 14 (Jul 26 2012), https://doi.org/10.1021/jm300687e.

[48] Sunhwan Jo et al., "CHARMM-GUI Membrane Builder for mixed bilayers and its application to yeast membranes," Biophysical journal 97, no. 1 (2009), https://doi.org/10.1016/j.bpj.2009.04.013. 
[49] E. L. Wu et al., "CHARMM-GUI Membrane Builder toward realistic biological membrane simulations," $J$ Comput Chem 35, no. 27 (Oct 15 2014), https://doi.org/10.1002/jcc.23702.

[50] M. A. Lomize et al., "OPM database and PPM web server: resources for positioning of proteins in membranes," Nucleic Acids Res 40, no. Database issue (Jan 2012), https://doi.org/10.1093/nar/gkr703.

[51] J. C. Phillips et al., "Scalable molecular dynamics with NAMD," J Comput Chem 26, no. 16 (Dec 2005), https://doi.org/10.1002/jcc.20289.

[52] W. Humphrey, A. Dalke, and K. Schulten, "VMD: visual molecular dynamics," J Mol Graph 14, no. 1 (Feb 1996), https://doi.org/10.1016/0263-7855(96)00018-5.

[53] Jing Huang and Alexander D MacKerell Jr, "CHARMM36 all-atom additive protein force field: Validation based on comparison to NMR data," Journal of computational chemistry 34, no. 25 (2013), https://doi.org/10.1002/jcc.23354.

[54] Kenno Vanommeslaeghe and Alexander D MacKerell Jr, "Automation of the CHARMM General Force Field (CGenFF) I: bond perception and atom typing," Journal of chemical information and modeling 52, no. 12 (2012), https://doi.org/10.1021/ci300363c.

[55] Jean-Paul Ryckaert, Giovanni Ciccotti, and Herman JC Berendsen, "Numerical integration of the cartesian equations of motion of a system with constraints: molecular dynamics of n-alkanes," Journal of computational physics 23, no. 3 (1977), https://doi.org/10.1016/0021-9991(77)90098-5.

[56] Tom Darden, Darrin York, and Lee Pedersen, "Particle mesh Ewald: An N · $\log (\mathrm{N})$ method for Ewald sums in large systems," The Journal of chemical physics 98, no. 12 (1993), https://doi.org/10.1063/1.464397.

[57] Scott E Feller et al., "Constant pressure molecular dynamics simulation: the Langevin piston method," The Journal of chemical physics 103, no. 11 (1995), https://doi.org/10.1063/1.470648.

[58] Michael L Connolly, "Analytical molecular surface calculation," Journal of applied crystallography 16, no. 5 (1983), https://doi.org/10.1107/S0021889883010985.

[59] Hui Liu and Tingjun Hou, "CaFE: a tool for binding affinity prediction using end-point free energy methods," Bioinformatics 32, no. 14 (2016), https://doi.org/10.1093/bioinformatics/btw215.

[60] N. A. Baker et al., "Electrostatics of nanosystems: application to microtubules and the ribosome," Proc Natl Acad Sci U S A 98, no. 18 (Aug 28 2001), https://doi.org/10.1073/pnas.181342398.

[61] R Core Team, "R: A language and environment for statistical computing," (Vienna, Austria, 2013).

[62] AN Shivanyuk et al., "Enamine real database: Making chemical diversity real," Chemistry today 25, no. 6 (2007).

[63] Roman A Laskowski and Mark B Swindells, "LigPlot+: multiple ligand-protein interaction diagrams for drug discovery," (ACS Publications, 2011). 


\section{Supplementary Information}

A computational binding affinity estimation protocol with maximum utilization of experimental data : A case study for Adenosine Receptor.

Il Kwon Cho a, Sung Hyun Moon a, and Kwang-Hwi Cho a,b, *

${ }^{\text {a }}$ School of Systems Biomedical Science, Soongsil University, Seoul, Republic of Korea.

${ }^{\mathrm{b}}$ AzothBio, Rm. 1306 MState A, 114, Beobwon-ro, Songpa-gu, Seoul, Republic of Korea

* Corresponding author. School of Systems Biomedical Science, Soongsil University, Seoul, Republic of Korea

E-mail addresses: chokh@ssu.ac.kr

\section{Table S1}

Components of A2aR membrane system

Component Atom

POPC 22914

Water

49482

A2AR

$\mathrm{Na}+$

43

Cl-

60

Total

79752 
(a)

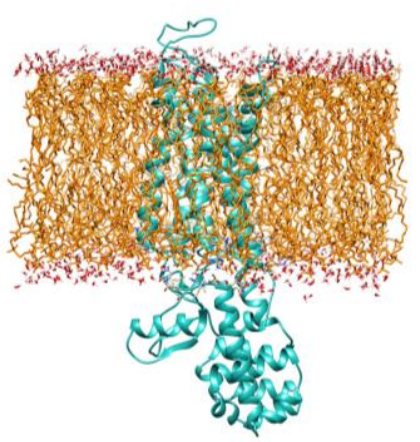

(b)

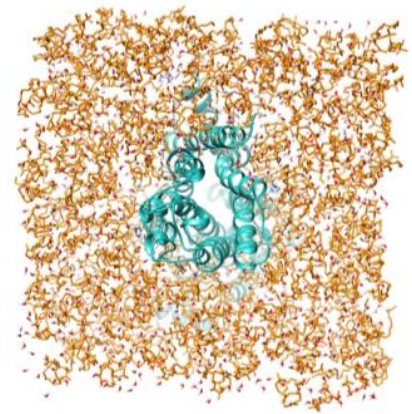

\section{Figure S1}

(a) Composition of POPC bilayer membrane system

(b) A2aR inserted in POPC bilayer 

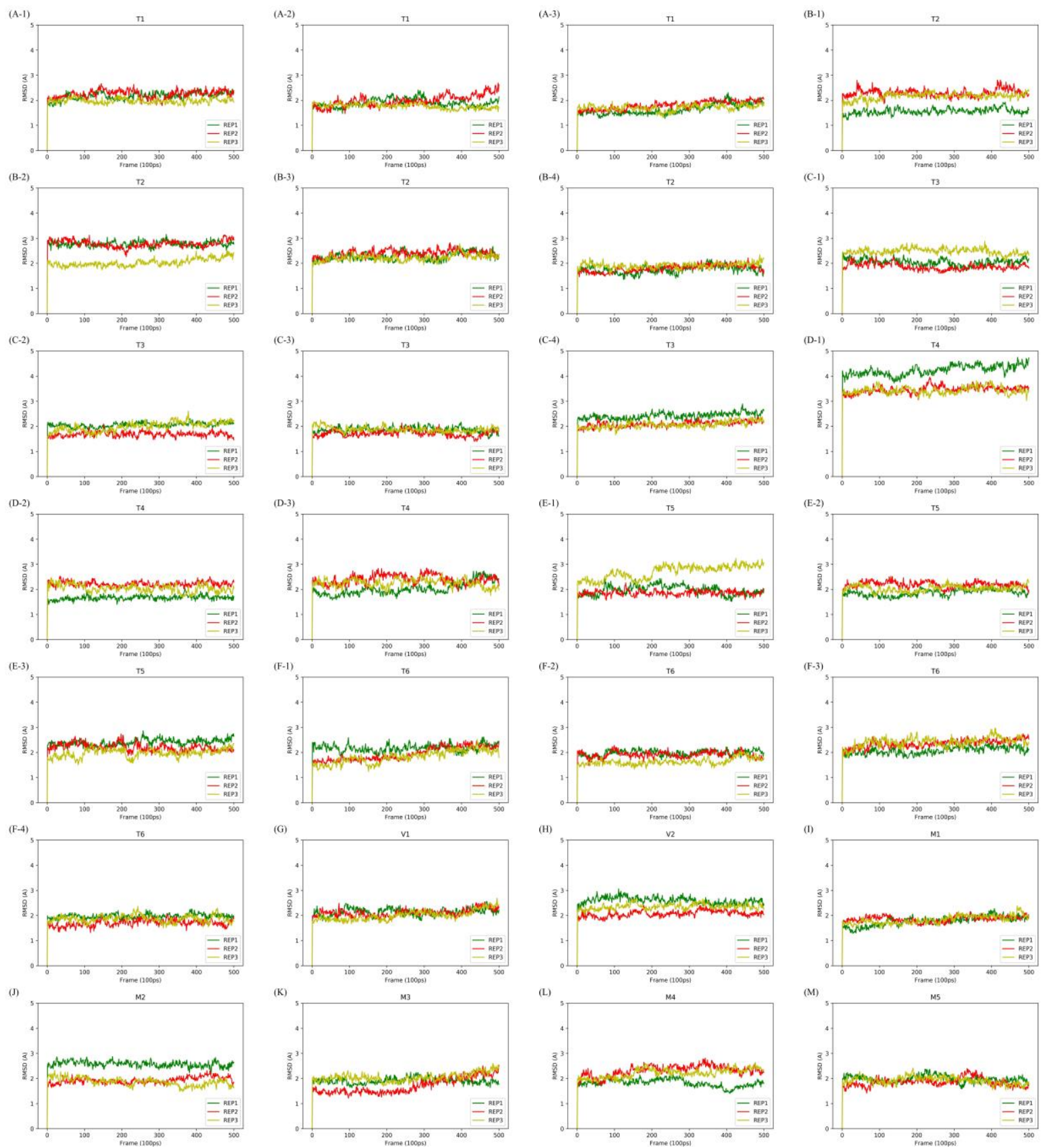

Figure S2

RMSD values of 500 frames ( $100 \mathrm{ps}$ / frame ) which were conducted in calculating binding energy. RMSD values of compound T1 to T6 were denoted as A to F, where three top-ranked poses with Vina and top-ranked poses with Smina were included. RMSD values of compound $V 1$ and $V 2$ were denoted as $G$ and $H$, and compound $M 1$ to $M 5$ were denoted as I to $M$. 


\section{Table S2}

MMPBSA energies of Compound T1 to T6 with three replicas for the protein-ligand poses from Vina and Smina. Vina was considered among three top-ranked poses and Smina was considered from top-ranked pose. Average binding energy among three top-ranked poses of Vina + MMPBSA energy, and top-ranked pose of Smina + MMPBSA were computed as $\Delta \mathbf{G}_{\text {mean }}$

\begin{tabular}{|c|c|c|c|c|c|c|}
\hline ID & $\begin{array}{c}\text { Vina+MM } \\
\text { PBSA } \\
\end{array}$ & Rank & $\Delta \mathrm{G}_{\text {mean }}$ & $\begin{array}{c}\text { Smina }+M M \\
\text { PBSA }\end{array}$ & Rank* $^{*}$ & $\Delta \mathrm{G}_{\text {mean }}$ \\
\hline \multirow[t]{15}{*}{$\mathrm{T} 1$} & -11.8538 & 1 & & -19.3815 & & \\
\hline & \pm 4.8515 & & & \pm 5.0353 & & \\
\hline & -17.1830 & & & & & \\
\hline & \pm 5.1485 & & & & & \\
\hline & $\begin{array}{l}-12.6784 \\
\pm 4.2961\end{array}$ & & & & & \\
\hline & $\begin{array}{l}-13.6680 \\
-1301 \\
-10\end{array}$ & 2 & -15.554 & -17.0155 & 3 & -19.1877 \\
\hline & \pm 6.0781 & & & \pm 4.7476 & & \\
\hline & $\begin{array}{l}-15.6768 \\
\pm 5.5820\end{array}$ & & & & & \\
\hline & $\begin{array}{l}-11.3624 \\
\pm 5.0235\end{array}$ & & & & & \\
\hline & -19.3815 & 3 & & -21.1662 & & \\
\hline & \pm 5.0353 & & & \pm 4.5937 & & \\
\hline & -17.0155 & & & & & \\
\hline & \pm 4.7476 & & & & & \\
\hline & -21.1662 & & & & & \\
\hline & \pm 4.5937 & & & & & \\
\hline \multirow[t]{18}{*}{$\mathrm{T} 2$} & -16.0879 & 1 & & -17.1005 & & \\
\hline & \pm 4.5151 & & & \pm 6.2413 & & \\
\hline & -9.8581 & & & & & \\
\hline & \pm 4.1269 & & & & & \\
\hline & -10.7023 & & & & & \\
\hline & \pm 4.4940 & & & & & \\
\hline & -15.0581 & 2 & -11.785 & -15.9373 & 4 & -17.2685 \\
\hline & \pm 8.0934 & & & \pm 4.5321 & & \\
\hline & -6.2853 & & & & & \\
\hline & \pm 4.1727 & & & & & \\
\hline & -11.1700 & & & & & \\
\hline & \pm 6.3831 & & & & & \\
\hline & -16.3252 & 3 & & -18.7676 & & \\
\hline & \pm 4.8337 & & & \pm 5.1203 & & \\
\hline & -8.9967 & & & & & \\
\hline & \pm 4.3973 & & & & & \\
\hline & -11.5815 & & & & & \\
\hline & \pm 4.9253 & & & & & \\
\hline \multirow[t]{7}{*}{$\mathrm{T} 3$} & -21.2512 & 1 & & -11.5522 & & \\
\hline & \pm 3.5536 & & & \pm 4.0499 & & \\
\hline & -16.3559 & & & & & \\
\hline & \pm 4.3237 & & & & & \\
\hline & -23.3313 & & & & & \\
\hline & \pm 2.8059 & & & & & \\
\hline & $\begin{array}{l}-16.3853 \\
\pm 3.0718\end{array}$ & 2 & -17.2124 & $\begin{array}{l}-13.7951 \\
\pm 5.2212\end{array}$ & 14 & -13.8012 \\
\hline
\end{tabular}




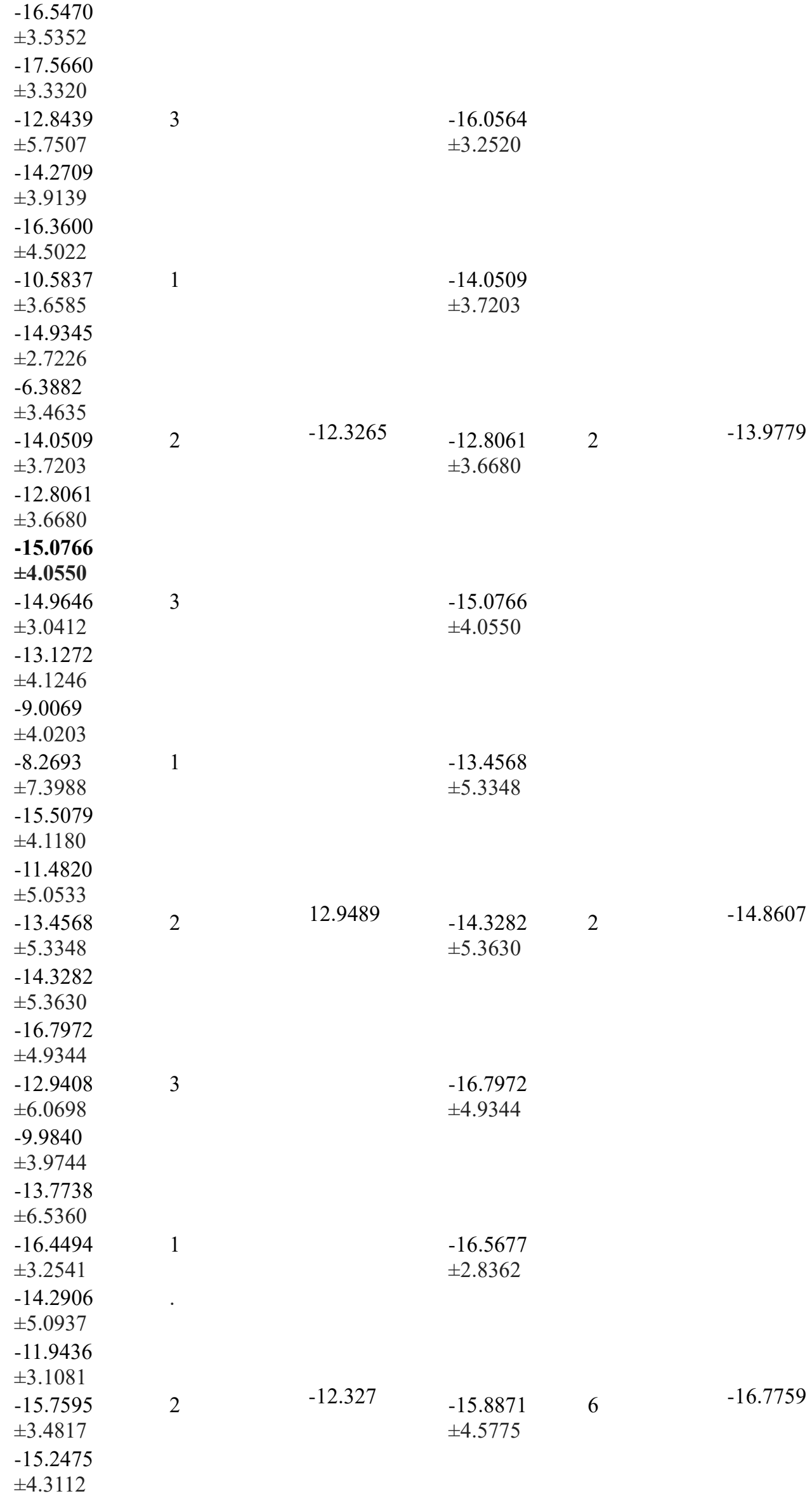

$-14.0509$

$\pm 3.7203$

$-15.0766$

$\pm 4.0550$

$-13.4568$

$\pm 5.3348$

2

12.9489

$-14.3282$

$\pm 5.3630$

2

$-14.8607$

$-16.7972$

$\pm 4.9344$

$-16.5677$

$\pm 2.8362$

2

$-12.327$

$-15.8871 \quad 6$

$-16.7759$ 
* denotes original Vina rank of top-ranked pose using Smina

\section{Table S3}

MMPBSA energies of Compound V1,V2 with three replicas for the best protein-ligand poses from Smina.

\begin{tabular}{llll}
\hline & \multicolumn{3}{c}{ Smina + MMPBSA } \\
\cline { 2 - 4 } ID & 1 & 2 & 3 \\
\hline V1 & -14.1847 & -16.8730 & -14.7824 \\
& \pm 3.8292 & \pm 2.9304 & \pm 3.4430 \\
& & & \\
\hline V2 & -11.5169 & -12.6115 & -17.3106 \\
& \pm 9.3034 & \pm 3.0578 & \pm 4.3883 \\
& & & \\
\hline
\end{tabular}

\section{Table S4}

MMPBSA energies of Compound M1 to M5 with three replicas for the best protein-ligand poses from Smina.

\begin{tabular}{llll}
\hline & \multicolumn{3}{c}{ Smina + MMPBSA } \\
\cline { 2 - 4 } ID & \multicolumn{3}{c}{3} \\
\hline M1 & -10.9705 & -4.7089 & -11.4454 \\
& \pm 2.5407 & \pm 3.4918 & \pm 2.8526 \\
& & & \\
\hline M2 & -16.1287 & -20.0426 & -17.0718 \\
& \pm 3.0615 & \pm 3.6762 & \pm 3.7639 \\
\hline M3 & & & -17.2859 \\
& & -19.1460 & \pm 3.2671 \\
& -18.9018 & \pm 3.4844 & -14.4564 \\
& \pm 3.3624 & & \\
\hline M4 & & -13.0945 & \\
& & \pm 3.4645 & \\
& & & \\
\hline
\end{tabular}


